CRL-JC-122525

PONF-95/0304--2

\title{
The Role of High Rydberg States in the Generation of Negative Ions in Negative-ion Discharges
}

\author{
J.R. Hiskes
}

This paper was prepared for submittal to

International Symposium of Negative Ion Production

Brookhaven, New York

October 23-27, 1995

November 28, 1995

This is a preprint of a paperintended forpublication in a joumal or proceedings. Since changes may be made before publication, this preprint is made available with the understanding that it will not be cited or reproduced without the permission of the author.

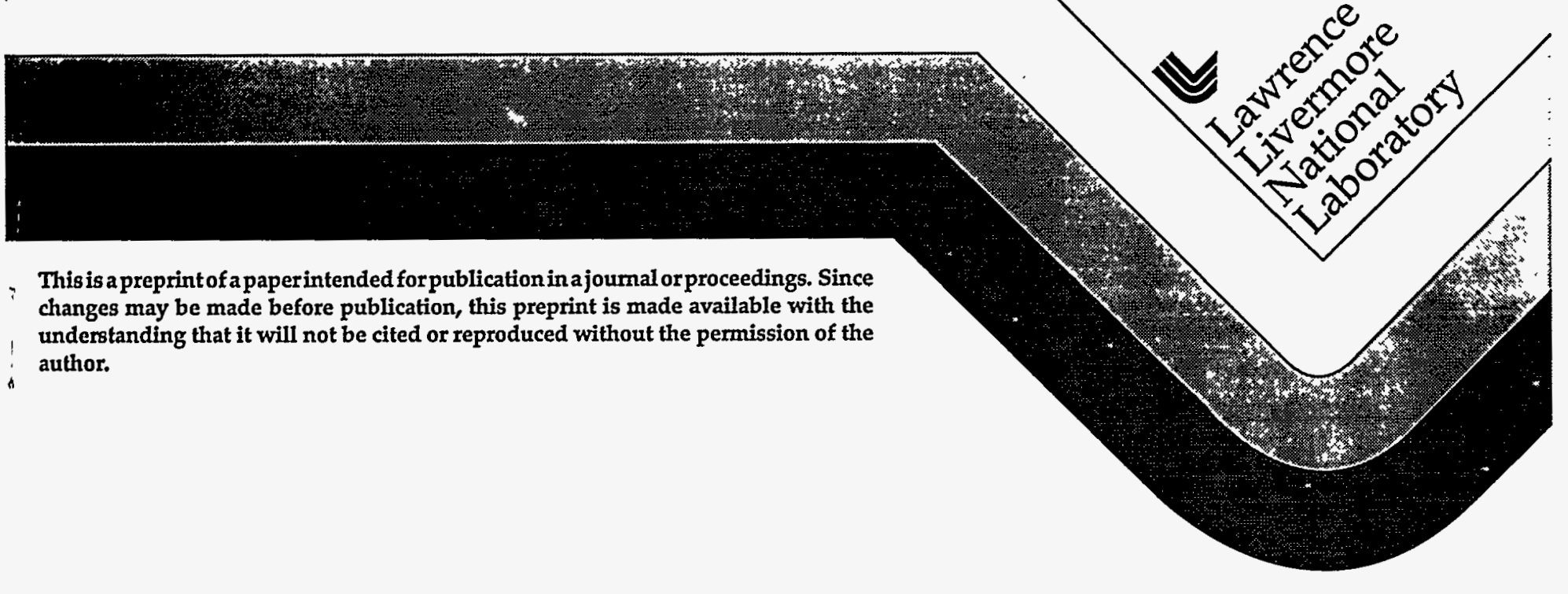




\section{DISCLAIMER}

This document was prepared as an account of work sponsored by an agency of the United States Government. Neither the United States Government nor the University of California nor any of their employees, makes any warranty, express or implied, or assumes any legal liability or responsibility for the accuracy, completeness, or usefulness of any information, apparatus, product, or process disclosed, or represents that its use would not infringe privately owned rights. Reference herein to any specific commercial product, process, or service by trade name, trademark, manufacturer, or otherwise, does not necessarily constitute or imply its endorsement, recommendation, or favoring by the United States Government or the University of California. The views and opinions of authors expressed herein do not necessarily state or reflect those of the United States Government or the University of California, and shall not be used for advertising or product endorsement purposes.

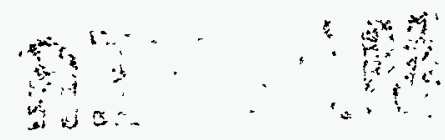




\title{
THE ROLE OF HIGH RYDBERG STATES IN THE GENERATION OF NEGATIVE IONS IN NEGATIVE-ION DISCHARGES
}

\author{
J. R. Hiskes \\ Lawrence Livermore National Laboratory
}

\begin{abstract}
The presence of high Rydberg states in a negative-ion discharge will increase the concentration of negative ions on the order of order of one per cent compared to those generated via dissociative attachment to ground-state vibrationally-excited molecules.
\end{abstract}

\section{INTRODUCTION}

The generation of substantial yields of $\mathrm{H}^{-}$ions in a laser excited $\mathrm{H}_{2}$ gas has been reported by Pinnaduwage and Christoforu.'

These $\mathrm{H}^{-}$yields have been attributed to $(2+1)$ REMP photoexcitation processes leading to dissociative attachment of doubly-excited or superexcited states (SES), or dissociative attachment of high Rydberg product states. The new feature of these experiments is the implied large dissociative attachment rates, of order $10^{-6} \mathrm{~cm}^{3} \mathrm{sec}^{-1}$, values that are orders-of-magnitude larger than the dissociative attachment of the vibrationally excited levels of the ground electronic state.

While these laser excitations are not directly applicable to a hydrogen negative-ion discharge, the implication of large dissociative attachment rates to the high Rydberg states may affect both the total negative-ion density and the interpretation of discharge performance. Within the discharge energetic electrons will collisionally excite the higher Rydberg states, and the relative contribution of the dissociative attachment of these states when compared with the dissociative attachment to the ground state vibrational levels, is the topic of this paper. 


\section{GENERATION OF THE RYDBERG POPULATION DENSITY}

The rate equation for the excitation of an electronic state $N_{R}$ of the $\mathrm{H}_{2}$ molecule is

$\alpha N_{R}(n) / d t=\eta_{f} N_{2} \sigma v(1-n)-N_{R}(n)\left\{\left[n_{f}[\sigma v(\dot{a})+\sigma v(e)]+n_{t h}[\sigma v(D A)+\sigma v(e)]+A_{H}\right\}(\mid A)\right.$ where $N(n), N_{2}, n_{p}$ and $n_{t h}$ are the densities of the excited electronic states with principal quantum number parentage $\Pi$, gas density, fast electron density and thermal electron density; the ov(1-n) is the electron excitation rate from the ground state to level n, ov(i), ov(e), ov)DA) are the ionization, excitation, and dissociative attachment rates, and $A_{n}$ the radiative decay rate. For the equilibrium solution, $\mathrm{dN} / \mathrm{dt}=0$, one has
$N_{R}(n)=\Pi_{f} N_{2} \dot{\sigma} v(1-n)\left\{n_{f}[\sigma v(i)+\sigma v(e)]+\Pi_{t h}[\sigma v(D A)+\sigma v(e)+\sigma v(i)]+A_{n}\right\}^{-1}(1 B)$ Here the solutions of $E_{\psi s} .1 \mathrm{~A}$, $1 \mathrm{~B}$ will be compared with the discharge data of Eenshuistra et al. ${ }^{3}$

The princi;al excitations are to the ${ }^{\prime} \Sigma_{g}{ }^{\prime} \Sigma_{\mu}$, and ${ }^{\prime} \prod_{\mu}$ states. For states $n<7,8$, the radiative term, $A_{n}$, dominates in the bracket. The equilibrium solution of $\mathrm{Eq}(1)$ becomes

$$
N_{R}(n)=H_{f} N_{2} \sigma v(1-n) A_{n}^{-1} \text {. }
$$

The excitation rates as a function of parentage quantum number, $\Pi$, are plotted in Fig. 1 for each of the sequences

$$
\eta p \sigma^{\prime} \sum_{\mu}, \mu p \pi^{\prime} T_{\mu} \text {, and } n s \sigma^{\prime} \sum_{i}
$$

and for incident electron energies of $40 \mathrm{eV}$. There is experimental data for states through $n=4$; for the higher Rydberg states these cross sections are extrapolated as $\mathrm{n}^{-3}$.

The radiative transition probabilities, $A_{n}$, are plotted in Fig. 2. Again, the transition probabilities beyond $n=3$ are scaled as $n^{-3}$. The ${ }^{\prime} \Sigma_{g}$ states are somewhat ambiguous. The E, F $\sum_{g}$ state has an anomolously small transition probability, since only the lower lying $B^{\prime} \Sigma_{\mu}$ state is accessible by radiative decay. For higher $\Sigma_{g}$ states both ${ }^{1} \Sigma_{\mu}$ and ${ }^{\prime} \pi_{\mu}$ states are accessible by radiative decay in analogy with the atomic transitions. For the $M S \sigma^{\prime} \Sigma_{g}$ states higher than the E, F state the equivalent atomic transition probabilities are used. 
Electronic states with Rydberg parentage equal to $n=3$ or higher ( $D^{\prime} \pi_{\mu} 3 p \pi$ and higher states) are subject to internal conversion which in the case of the $\mathrm{H}_{2}$ molecule manifests itself through. autoionization,

$$
H_{2}^{*}(n) \rightarrow H_{2}^{+}+e
$$

provided the vibrational level, $v$, of the state in question lies above the ground vibrational state of the $\mathrm{H}_{2}^{+}$ion. For these Rydberg states the internuclear potential functions closely approximate that of the $\mathrm{H}_{2}^{+}$ion, and the vibrational population fractions, $f(v)$, calculated for the ionization of $\mathrm{H}_{2}$ to form $\mathrm{H}_{2}^{+}{ }^{4}$, can be used to approximate the $\mathrm{H}_{z}^{*}(\mathrm{v})$ populations.

The fraction of Rydberg excitations, $F(n)$ that survives autoionization is

$$
F(n)=\sum_{v} f(v) \text {, }
$$

where the upper limit on $v$ is the highest level that lies below the $\mathrm{v}=0$ level of $\mathrm{H}_{2}^{+}$. This limit is obtained by inspection of Sharp's diagram. The values for $F(n)$ are given in Table I. The ${ }^{\prime} \Sigma_{g}$ values are taken equal to the ' $\Sigma_{\mu}$ values.

\begin{tabular}{|c|c|c|}
\hline n & State & $F(n)$ \\
\hline \multirow[t]{2}{*}{2} & $\mathrm{~B}^{\prime} \Sigma_{\mathrm{N}}$ & 1. \\
\hline & $c^{\prime} \pi_{2}$ & 1. \\
\hline \multirow[t]{2}{*}{3} & $\mathrm{~B}^{\prime \prime} \Sigma^{n}$ & 1. \\
\hline & $\mathrm{D}^{\prime} \pi_{\mu}$ & .84 \\
\hline \multirow[t]{2}{*}{4} & $\mathrm{~B}^{\prime \prime}{ }^{\prime}{ }^{\mathrm{N}}$ & .68 \\
\hline & $\mathrm{D}^{\prime \prime} \pi_{\mu}$ & .57 \\
\hline \multirow[t]{2}{*}{5} & $B^{\prime \prime \prime}{ }^{\prime} \Sigma$ & .41 \\
\hline & $D^{\prime \prime} \prime \pi$ & .41 \\
\hline \multirow[t]{2}{*}{6} & $B^{\prime \prime \prime \prime \prime} \sum^{n}$ & .25 \\
\hline & $D^{\prime \prime \prime} T^{\prime \prime}$ & .25 \\
\hline \multirow[t]{2}{*}{7} & $B^{*+1} 1 \sum^{n}$ & .09 \\
\hline & & .09 \\
\hline
\end{tabular}

\section{TABLE I}


States $\mathrm{n}=7$ and higher have only their ground vibrational level lying below the ground level of $\mathrm{H}_{2}^{+}$, and for these states all $F(n)=.09$. The ov $(1-n)$ in Eq. 2A is replaced with $F(n)$ ov $(1-n)$.

Above the state $n=7$ the collisional-radiative process is changed in a qualitative way. The radiative decay of the $n=7$ state is offset by the larger collisional excitation rate to $n=8$ state. These $\Delta M=1$ excitations increase as $\mathrm{n}^{4}$, specifically

$\sigma(M, n+1)=\sigma_{0} \mu^{4}$,

with $\sigma_{0}=3 \times 10^{-16} \mathrm{~cm}^{2}$. The rapid increase of this collision processes at successively higher states causes a runaway cascade terminated by ionization at the highest bound state sustainable in the discharge environment.

The steady-state rate equations for an array of states beginning at $n=7$ is solved taking into account the array of collision processes given by (5) and

From these solutions one obtains

$$
\sigma(n+1, n)=\sigma_{0} n^{4}
$$

$$
N_{R}(7+k) / N_{R}(\gamma)=\left(\frac{1}{2}\right)^{k}, k=1,2,3, \ldots
$$

i.e., the $n=8$ level has one-half the population of $n=7$, the $n=9$ level one-fourth the population, etc.

One can sum the populations of all states above $n=7$,

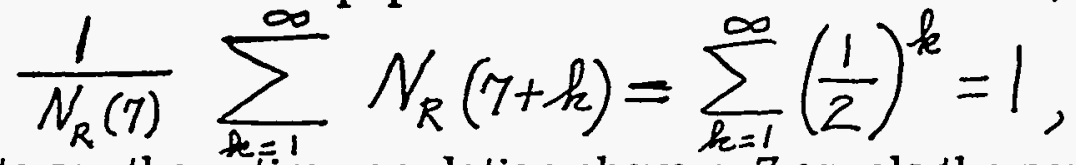

that is to say the entire population above $n=7$ equals the population of the $n=7$ state.

The cross sections for excitation of the triplet states at $40 \mathrm{eV}$ are only five to fifteen percent of the singlet excitations, but with comparable radiative transition probabilities. An exception is the $2 p \pi c^{3} \pi_{\mu}$ state with $A=10^{4} \mathrm{sec}^{-1}$. This state is collisionally excited to the rapidly decaying and close lying $a^{3} \sum_{g}$ state in a time $\simeq 8$ $\mu \mathrm{sec}$ to give an effective transition probability $A\left(3 \pi_{\mu}\right) \simeq 1.2 \times 10^{6} \mathrm{~s}^{-1}$ The solutions for the Rydberg populations, $\mathrm{N}_{\mathrm{R}}(n) \mathrm{mol} . \mathrm{cm}^{-3}$, are obtained from Eqs. 1 and plotted in Fig. 3. Here we have increased the populations by ten percent to account for the triplet excitations. 
These population distributions are shown in two approximations:

In the lower limit case only the population of the $n=7$ state originating from the $\sigma(1-7)$ excitation is included in the upward cascade. In the upper limit all higher excitations given by the sum $\sum_{k=1}^{\infty} \sigma(1 \rightarrow k)$ are included for excitations initiated at the $n=7$ level.

\section{GENERATION OF THE NEGATIVE ION DENSITY}

The negative ion density generated by each Rydberg state is given by

$N_{R}^{-}(M)=n_{t h} N_{R}(H) \sigma v(D A)\left\{n_{t h}\left[\sigma v(C D+\sigma v(i i)]+N_{1} \sigma v(A D)\right\}^{-1}\left[1-e^{-D t}\right],(q)\right.$ where the successive collision terms in the denominator, $D$, are electron collisional detachment, ion-ion neutralization, and associative detachment by collisions with atoms, $N_{1}$. The total negative ion concentration is

$$
N^{-}=\sum_{n=1}^{\infty} N_{R}(H) .
$$

In Fig. 4 are plotted the negative ion concentrations as a function of discharge electron temperature taken from the FOM experiment. ${ }^{3}$ The error bars have been reported by Hopman. 7

At the previous Symposium in this series the author reported $^{8}$ the calculation of the ion density at $\mathrm{kT}=1.4 \mathrm{eV}$, using the numerical negative ion code and assuming dissociative attachment to the vibrational levels, $\mathrm{H}_{2}(\mathrm{~N})$, belonging to the ground state. In this earlier calculation only those solutions for the $\mathrm{H}_{2}(v)$ that fell within the experimentally observed range were retained for the calculation of the negative ion density. The result of this calculation at $k T=1.4 \mathrm{eV}$ is shown by the trianjle the figure. These calculations have now been extended to the lower electron temperatures and fall within the range of the experimental uncertainties.

Ignoring the ground state $\mathrm{H}_{2}(\mathrm{w})$ contributions but including the Rydberg contribution given by Eq. 10, the upper and lower limit calculations are shown on the lower part of the figure. Comparing, the $\mathrm{H}_{2}(v)$ contribution to the negative ion density is approximately fifty times larger than the Rydberg contribution. 
The group at the Wright Laboratories ${ }^{1 /}$ have also performed calculations for the negative ion density including only contributions from the Rydberg states and taking into account only the collisional excitation, $\sigma(1-n)$, and the subsequent radiative decay. Their solutions are shown by the circles in Fig. 4, and are seen to be approximately a factor of a hundred larger than those reported here.

This large discrepancy is readily understood if we examine the approximations made by the Wright group. Their model for the Rydberg density assumes a single effective state with excitation cross section equal to $2.4 \times 10^{-17} \mathrm{~cm}^{2}$ and a radiative decay rate of $10^{6} \mathrm{sec}^{-1}$ to give $\sigma / A=2.4 \times 10^{-23}\left(\right.$ cf $\left.\varepsilon_{q .} 2 A\right)$, In our calculation the principal contribution to the excitation is to levels $n=3,4$. For these states the contribution from ${ }^{\prime} \Sigma_{g}{ }^{\prime} \Sigma_{\mu},{ }^{\prime} \prod_{\mu}$ gives $\sigma / A \simeq 2.2 \times 10^{-25}$, and the factor of a hundred is recovered.

\section{HIGH DENSITY SYSTEMS}

Consider next the extrapolation to high-density, high-power systems. The relative contributions to the ion density are given by the relative values of the vibrational population and the Rydberg population, respectively. The vibrational population is equal, roughly speaking, to

$$
\begin{aligned}
& N_{2}(v)=\frac{M_{f} N_{2} \sigma v(E-v)}{\left[n_{f} \sigma v(e)+n_{t h} \sigma v(d i s s)\right.}\left[1-e^{-D t}\right],(I) \\
& \text { and the Ryd berd population to } \\
& N_{R}(M)=\frac{n_{+} N_{2} \sigma v(1-n)}{\left[A_{m}+n_{t h} \sigma v(e)\right]}\left[1-e^{-D t]},\right. \text { (12) }
\end{aligned}
$$

Both expressions have the same density factors in the numerator. Both denominators are sensitive to the electron density, but the Rydberg denominator has a balance between excitation and radiative decay. Until the specific level or levels are known that express 
the large dissociative attachment rates, the scaling with density is obscured by the radiative decay term. In the above it has been assumed that the large rates have their onset at $n=3$. If the onset is at $n=5$, for exmple, the ratio above for the FOM data is increased a factor of ten to five-hundred to one. But at $n=5$ the collisional term dominates the $A_{5}$ radiative term at electron densities a factor of four or more higher; and at these higher densities the ratio is again restored to approximately one hundred. The scaling of the ratio of the vibrational to Rydberg contributions with increased electron density will not be clarified precisely until the onset level for large dissociative attachment rates is known.

\section{SUMMARY}

The relative contributions of the high Rydberg states compared to the ground state vibrational excitation is of the order of one percent.

This work performed at LLNL under the auspices of the US DOE under Contract No. W-7405-ENG-48.

\section{FIGURE CAPTIONS}

Fig.1 Electron excitation rates versus parentage principal quantum number, $n$, for the ${ }^{1} \Sigma_{\mathrm{g}^{\prime}}{ }^{1} \sum_{\mathrm{u}}$, and ${ }^{1} \Pi_{\mathrm{u}}$ sequences.

Fig.2 The radiative transition probabilities versus principal quantum number, $n$, for the ${ }^{1} \Sigma_{\mathrm{g}^{\prime}}{ }^{1} \Sigma_{\mathrm{u}}$, and ${ }^{1} \Pi_{\mathrm{u}}$ sequences.

Fig.3 Rydberg populations, mol. $\mathrm{cm}^{-3}$ versus principal quantum number, $n$, for the lower limit and upper limit approximations.

Fig.4 Negative ion density versus discharge electron temperature: closed squares, Ref. 3; triangles, Refs. 8, 9; lower upper-lower limit symbols, these calculations; open circles, Ref. 10. 


\section{REFERENCES}

1. L.A. Pinnaduwage and L.G. Christophorou, Phys. Rev. Lett. $\underline{70}$, 754 (1993).

2. L.A. Pinnaduwage and L.G. Christophorou, J. Appl. Phys. $\underline{76}$, 46 (1994).

3. P.J. Eenshuistra, R.M.A. Heeren, A.W. Kleyn, and H.J.

Hopman, Phys. Rev. A느, 3613 (1989).

4. S. Rothenberg and E.R. Davidson, J. Mol. Spectrosc. 22, I (1967).

5. T.E. Sharp, Atomic Data, 2, 119 (1971).

6. J.R. Hiskes, Phys. Rev. Lett. 10. 102 (1963).

7. H.J. Hopman, Private Communication.

8. J.R. Hiskes, AIP Conf. Ser 287, p. 191, Nov. 1992.

9. J.R. Hiskes, to be published.

10. A. Garscadden and R. Nagpal, Plasma Sources Sci. Technol. 4. 268 (1995). 
Excitation Cross Section $\sigma(1 \rightarrow n)$

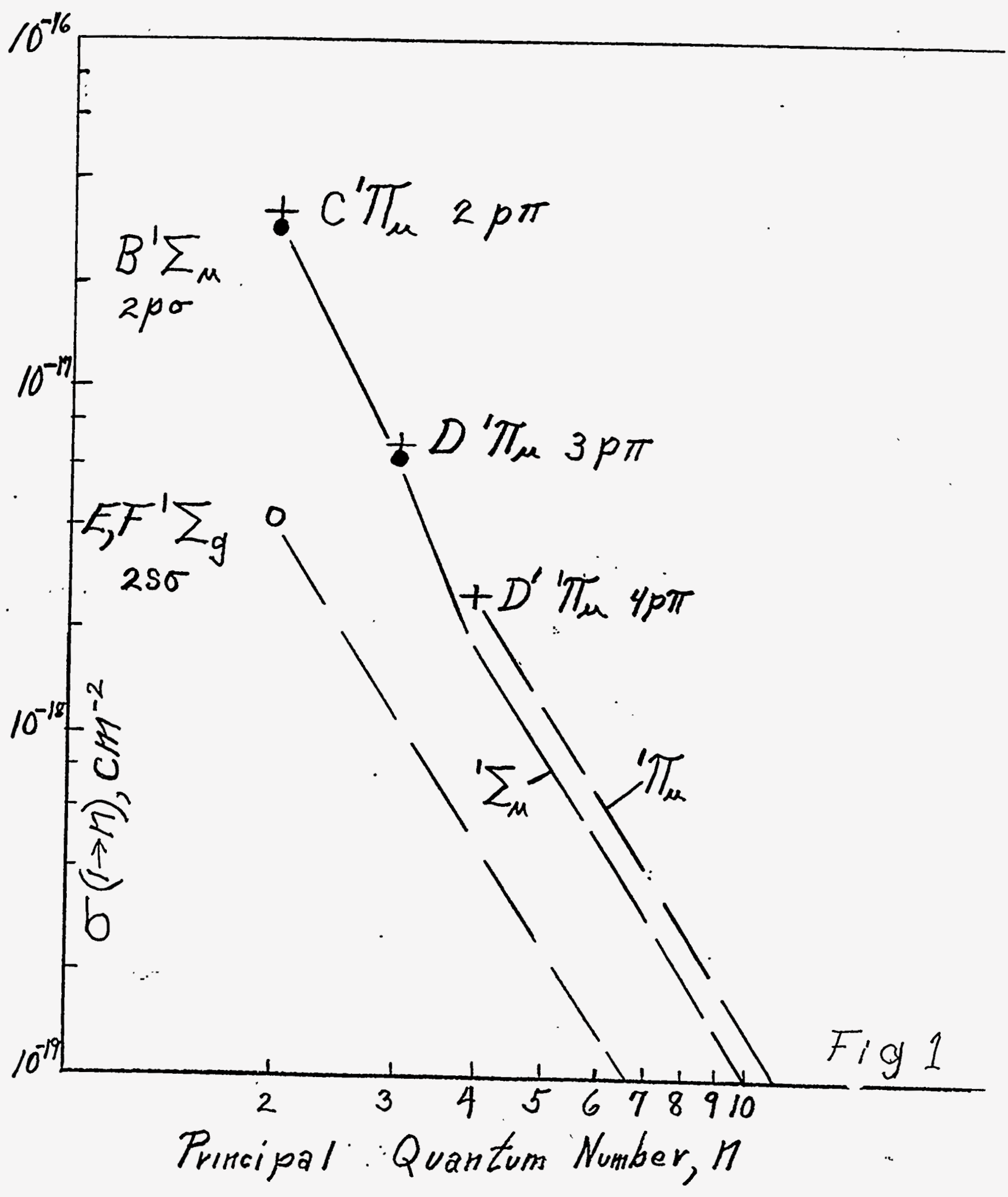


Radiative Transition Probabilities, $A_{n}$

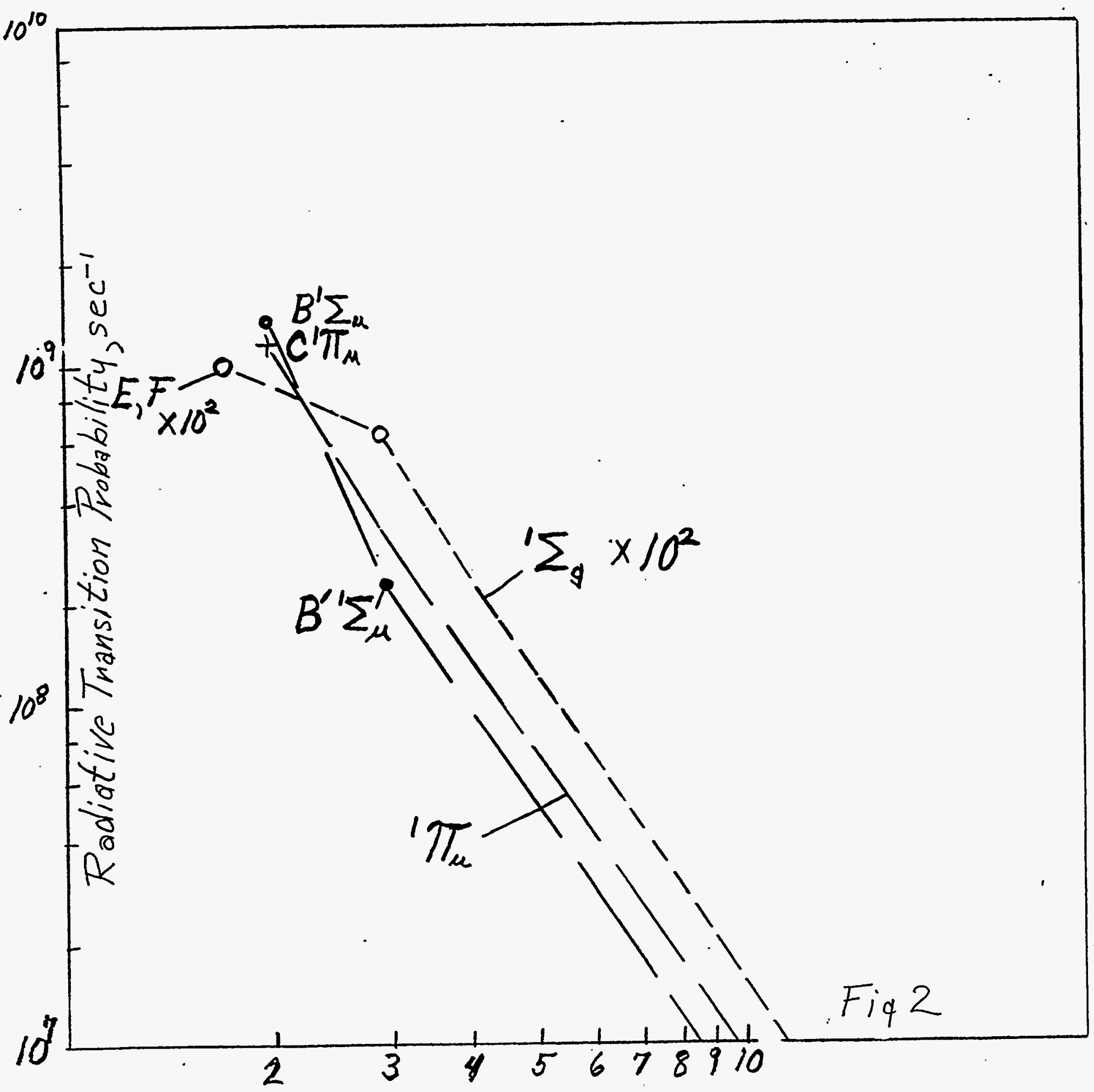

Principal Quantum Number, $n$ 


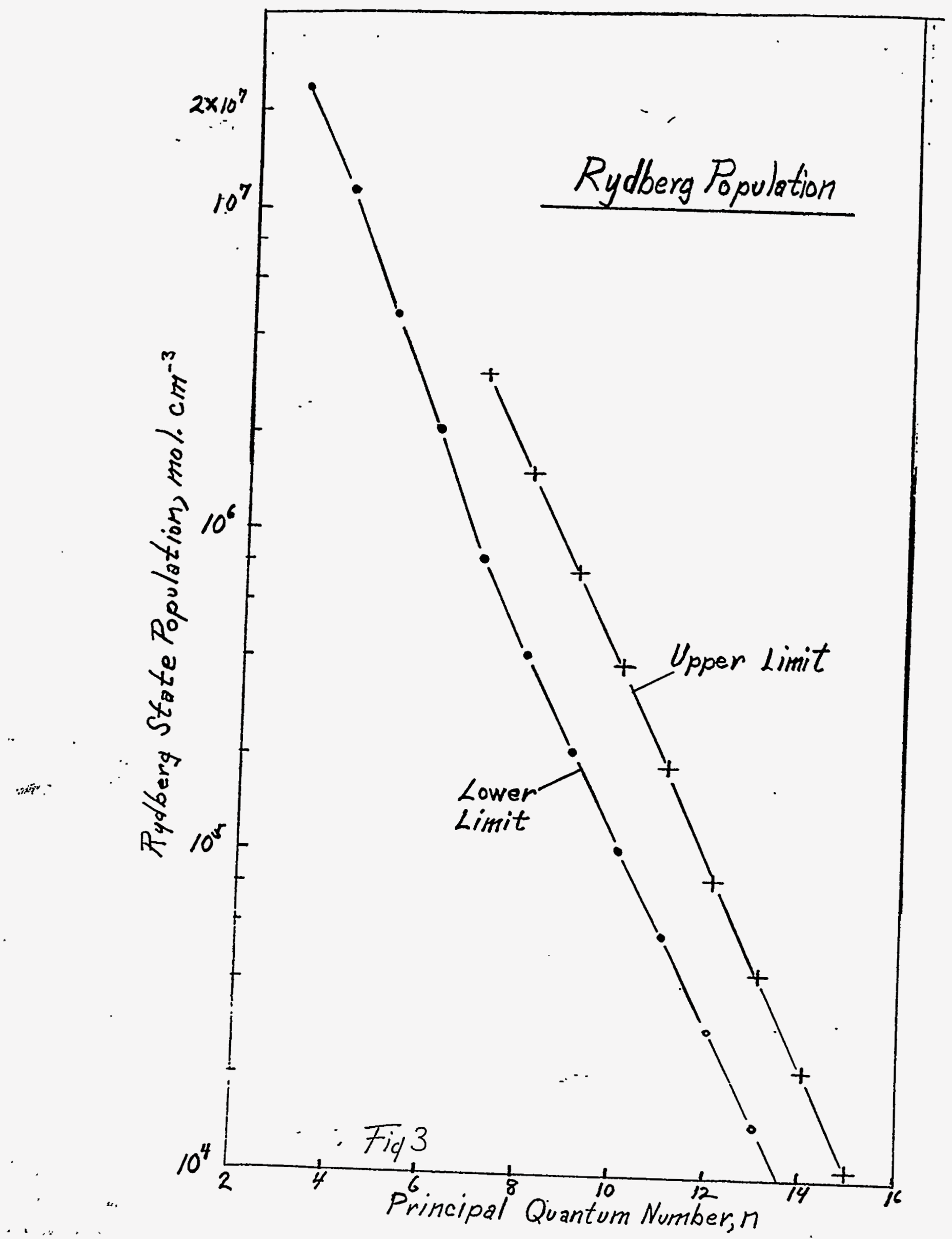




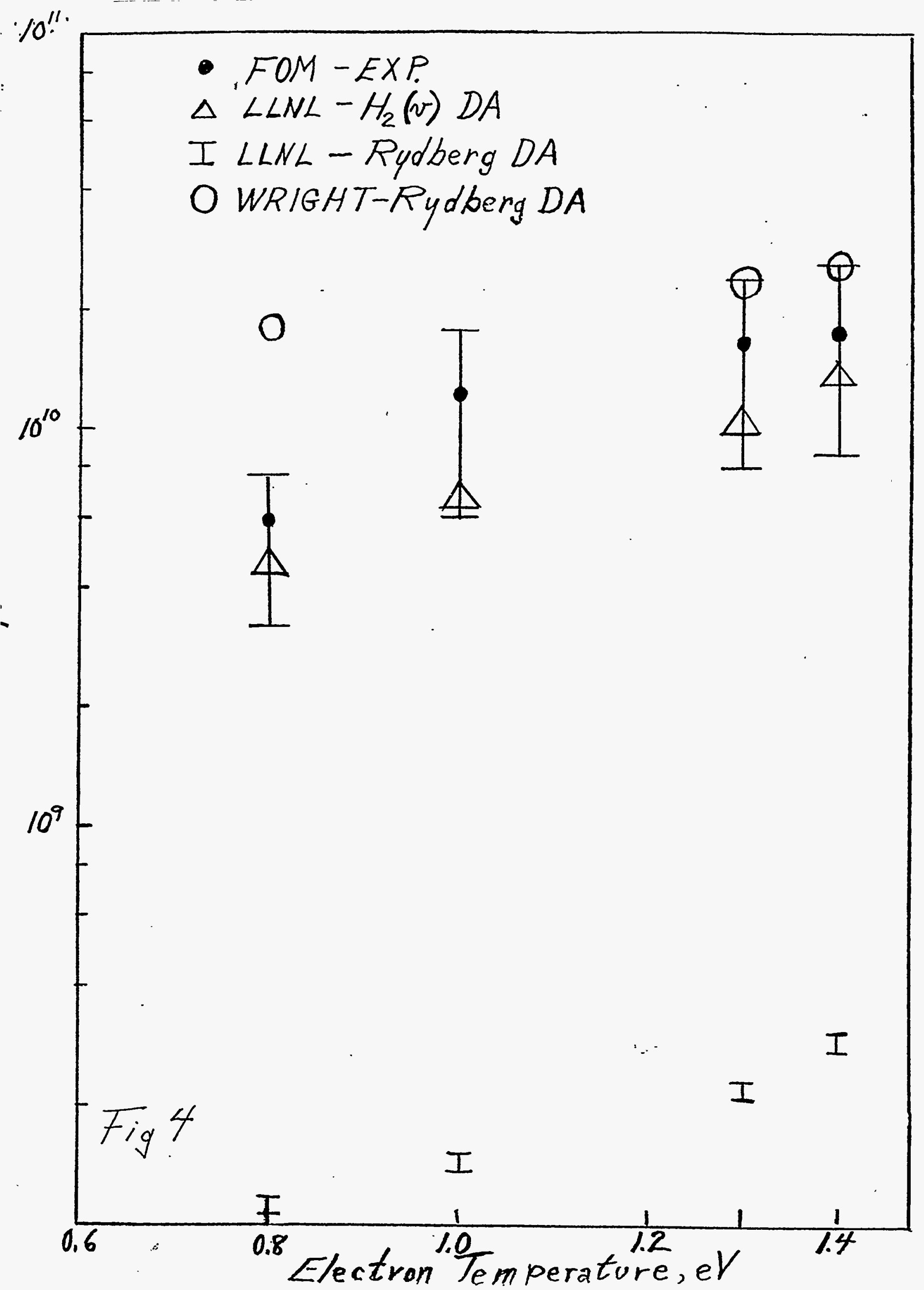




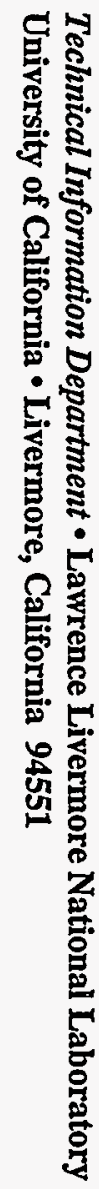

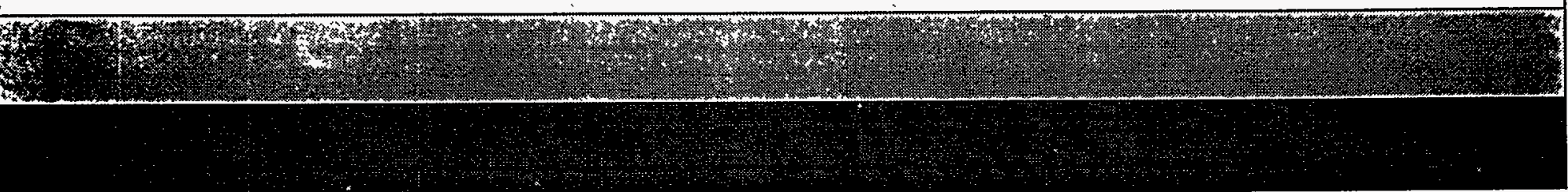

